\title{
The SarcoEndoplasmic Reticulum Calcium ATPase (SERCA) pump: a potential target for intervention in aging and skeletal muscle pathologies
}

\author{
Hongyang $\mathrm{Xu}^{1}$ and Holly Van Remmen ${ }^{1,2,3^{*}}$
}

\begin{abstract}
As a key regulator of cellular calcium homeostasis, the Sarcoendoplasmic Reticulum Calcium ATPase (SERCA) pump acts to transport calcium ions from the cytosol back to the sarcoplasmic reticulum (SR) following muscle contraction. SERCA function is closely associated with muscle health and function, and SERCA activity is susceptible to muscle pathogenesis. For example, it has been well reported that pathological conditions associated with aging, neurodegeneration, and muscular dystrophy (MD) significantly depress SERCA function with the potential to impair intracellular calcium homeostasis and further contribute to muscle atrophy and weakness. As a result, targeting SERCA activity has attracted attention as a therapeutical method for the treatment of muscle pathologies. The interventions include activation of SERCA activity and genetic overexpression of SERCA. This review will focus on SERCA function and regulation mechanisms and describe how those mechanisms are affected under muscle pathological conditions including elevated oxidative stress induced by aging, muscle disease, or neuromuscular disorders. We also discuss the current progress and therapeutic approaches to targeting SERCA in vivo.
\end{abstract}

Keywords: Skeletal muscle, SERCA, Calcium homeostasis, Aging, Neuromuscular disorder, Oxidative stress

\section{The role of SERCA in skeletal muscle metabolism}

Skeletal muscle is the largest organ in the body, contributing close to $40 \%$ of total body mass. It plays a major role in metabolism and key physiological and biochemical processes in addition to its critical functions of force generation and movement [25]. Contractile force generation is a process mediated by calcium ions resulting in the activation of interaction between myosin and actin filaments [30]. As a result, the regulation of calcium homeostasis is critical to proper maintenance of muscle function. A key regulator of cellular calcium

*Correspondence: Holly-VanRemmen@omrf.org

${ }^{1}$ Aging \& Metabolism Research Program, Oklahoma Medical Research Foundation, Oklahoma City, OK 73104, USA

Full list of author information is available at the end of the article homeostasis is the Sarcoendoplasmic Reticulum Calcium ATPase (SERCA) pump which acts to transport calcium ions from the cytosol back to the sarcoplasmic reticulum (SR) following muscle contraction. The SERCA protein is localized on the SR membrane and has been reported to be the most abundant protein in the SR [6].

The SERCA pumps belong to the family of P-type ATPases that includes a series of membrane-bound ATPases, such as plasma membrane $\mathrm{Ca}^{2+}$ ATPase (PMCA), $\mathrm{Na}^{+} / \mathrm{K}^{+}$ATPase, and $\mathrm{H}^{+} / \mathrm{K}^{+}$ATPase [64]. A common feature of these P-type ATPases is to transport metal ions against the gradient across the SR membrane coupled with the hydrolysis from ATP to ADP $[47,48]$. As illustrated in Fig. 1, the primary function of SERCA is the uptake of cytosolic $\mathrm{Ca}^{2+}$ back into SR lumen using energy derived from the hydrolysis of original author(s) and the source, provide a link to the Creative Commons licence, and indicate if changes were made. The images or other third party material in this article are included in the article's Creative Commons licence, unless indicated otherwise in a credit line to the material. If material is not included in the article's Creative Commons licence and your intended use is not permitted by statutory regulation or exceeds the permitted use, you will need to obtain permission directly from the copyright holder. To view a copy of this licence, visit http://creativecommons.org/licenses/by/4.0/. The Creative Commons Public Domain Dedication waiver (http://creativeco mmons.org/publicdomain/zero/1.0/) applies to the data made available in this article, unless otherwise stated in a credit line to the data. 


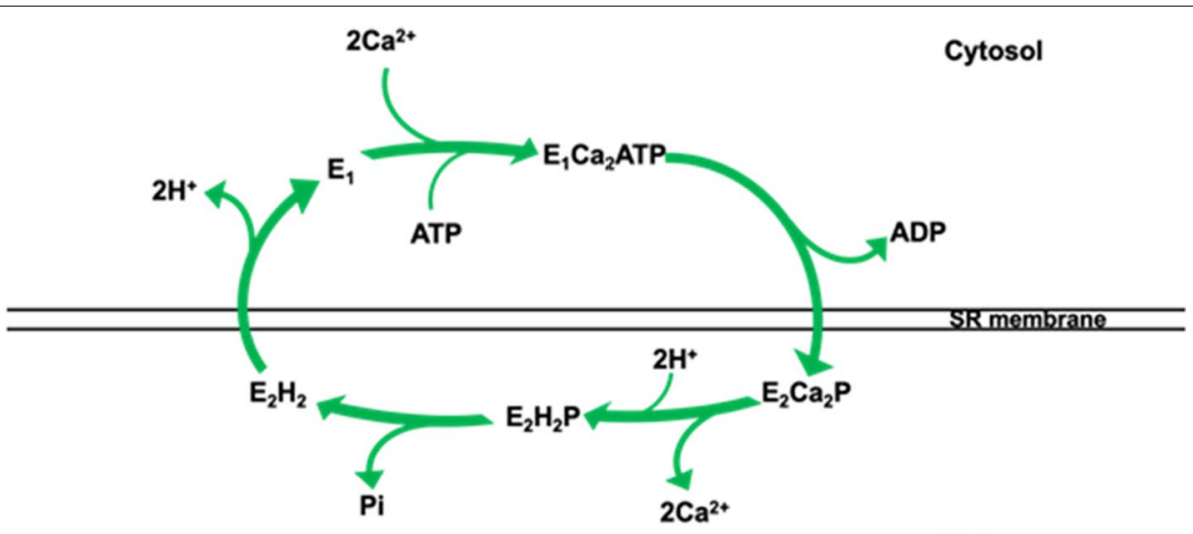

\section{SR Lumen}

Fig. 1 Schematic diagram of the kinetic cycle of SERCA pump. During the transportation of $\mathrm{Ca}^{2+}$ ions, ATP binding is coupled with the change of conformational states of SERCA from $E_{1}$ to $E_{2}$. These two states have different affinity to $\mathrm{Ca}^{2+}$ ions, with $\mathrm{E}_{1}$ being highly affinitive and $\mathrm{E}_{2}$ being low. Each time, there are two $\mathrm{Ca}^{2+}$ ions and an ATP molecule binding to $\mathrm{E}_{1}$ state $\left(\mathrm{E}_{1} \mathrm{Ca}_{2} \mathrm{ATP}\right)$, then after the hydrolysis of ATP the state of SERCA is changed to $E_{2}\left(E_{2} C a_{2} P\right)$ with the release of $A D P$. The release of $\mathrm{Ca}^{2+}$ ions into the SR lumen requires the exchange of luminal protons on $E_{2}$ state $\left(\mathrm{E}_{2} \mathrm{H}_{2} \mathrm{P}\right)$. The final step is dephosphorylation and dehydrogenation to return the enzyme to the ground state, $\mathrm{E}_{1}$, which then is able to initiate a new transport cycle. Figure reproduced based on Periasamy et al. 2007 [51]

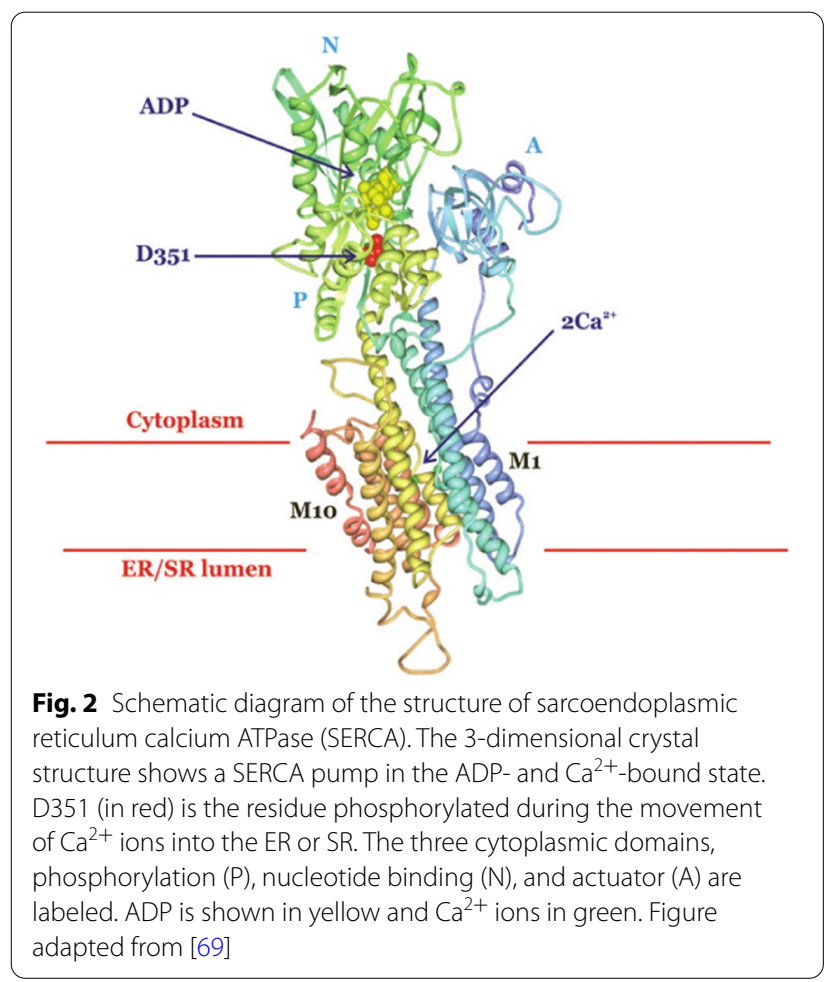

Fig. 2 Schematic diagram of the structure of sarcoendoplasmic reticulum calcium ATPase (SERCA). The 3-dimensional crystal structure shows a SERCA pump in the ADP- and $\mathrm{Ca}^{2+}$-bound state. D351 (in red) is the residue phosphorylated during the movement of $\mathrm{Ca}^{2+}$ ions into the ER or SR. The three cytoplasmic domains, adapted from [69]
ATP. This allows the maintenance of the cytosolic $\mathrm{Ca}^{2+}$ concentration at low levels between 50 and $100 \mathrm{nM}$ [5]. Figure 2 shows the structure of the SERCA pump as reported by Watson in 2015 [69], revealing a globular lobe that protrudes into the cytosol connecting with the SR membrane through a stalk that has only a minor extension into the lumen of the SR [5].

During excitation-contraction (E-C) coupling events associated with muscle force generation, $\mathrm{Ca}^{2+}$ ions are released through the ryanodine receptor (RyR) channels in the SR membrane increasing the cytosolic $\left[\mathrm{Ca}^{2+}\right]$ to $1-2 \mu \mathrm{M}$ for a few milliseconds. This high concentration of $\mathrm{Ca}^{2+}$ ions facilitates the interaction of calcium with troponin to trigger the sequence of events leading to force production [33]. However, prolonged high cytosolic $\left[\mathrm{Ca}^{2+}\right]$ can be detrimental to cellular homeostasis, stimulating calcium signaling pathways and leading to activation of proteases such as calpains [44] and matrix metalloproteinases (MMPs) that can degrade cellular components [58]. Moreover, calcium ions can directly control contractile function in muscles, especially in cardiac muscle, where the intracellular calcium release from the SR is triggered by a small influx of calcium, which is termed as calcium-induced calcium release (CICR). Elevated dysregulated calcium concentration can directly contribute to adverse cardiac remodeling and disruption of systolic and diastolic function [36]. Thus, dysfunctional SERCA pumps could contribute to high cytosolic calcium, limiting not only muscle function, but also causing impairments in cellular metabolism and function. 
The primary goal of this review is to provide an overview of the impact of pathological conditions such as high oxidative stress induced by aging, muscle disease or neuromuscular disorders on SERCA function, and the potential therapeutic approaches via targeting SERCA.

\section{SERCA pump isoforms and their regulation}

The SERCA pump family is encoded by three different genes, SERCA 1, SERCA 2, and SERCA 3, and seven different isoforms are expressed from these genes, SERCA 1a/1b, SERCA 2a/2b, and SERCA 3a/3b/3c [51] (Table 1). SERCA 1 is a skeletal muscle specific isoform expressed predominantly in fast-twitch muscles, with $1 \mathrm{a}$ and $1 \mathrm{~b}$ being adult and neonatal forms respectively. SERCA 2a is primarily expressed in slow-twitch skeletal muscle and cardiac muscle, while SERCA $2 b$ is a ubiquitous isoform appearing in all cell types at a low abundance. SERCA 3 is very rare in muscle tissues but is universally expressed in non-muscle tissues (i.e., neuronal cells and epithelial cells) at a very low level [52]. The structure of SERCA pumps is highly conserved despite the fact that they are encoded from several different genes, with SERCA 1 showing $84 \%$ similarity to SERCA $2 a$ and $75 \%$ to SERCA 3 . Because of their similar structures, it has been predicted that all SERCA isoforms might have similar native transmembrane arrangements and tertiary conformations, and hence, their sensitivity to $\mathrm{Ca}^{2+}$ and enzyme activity should be equivalent [38]. In particular, the muscle isoforms SERCA 1 and SERCA 2a have enzymatic properties that are almost identical [51].

The activity of the SERCA pumps is regulated by a series of small molecular weight proteins present in muscle, including phospholamban (PLN), sarcolipin (SLN), the dwarf open reading frame (DWORF), and the recently identified myoregulin (MLN) [4, 39, 46, 61]. All these regulatory proteins are expressed differentially in muscle; however, PLN, SLN, and the newly discovered MLN share a highly conserved hydrophobic motif in the transmembrane region which is rich in leucine residues, providing an interaction surface for binding to SERCA. PLN

Table 1 SERCA isoforms and its distribution in mammalian tissues

\begin{tabular}{ll}
\hline Cell type & SERCA isoforms \\
\hline Skeletal muscle & \\
Fast twitch & SERCA 1a, 1b*, 2a, 2b (*fetal only) \\
Slow twitch & SERCA 2a, 2b \\
Cardiac muscle & SERCA 2a, 2b \\
Non-muscle cells & SERCA 2b, 3a, 3b, 3c \\
\hline
\end{tabular}

is found to be expressed in all muscle types, but primarily interacts with the SERCA 2a isoform in cardiac and slowtwitch muscles. Compared to PLN, SLN can regulate both SERCA 1 and 2 isoforms [51]. Both PLN and SLN are found to be less effective on regulating SERCA 3 isoform, whereas in contrast, DWORF is found to be able to bind and regulate all three isoforms of SERCA, including SERCA 3 [46]. The physiological role of PLN in cardiac muscle and SLN in skeletal muscle has been well established with similar inhibitory effects on SERCA activity, but through different mechanisms. PLN inhibits SERCA activity by reducing the SERCA pumps affinity to $\mathrm{Ca}^{2+}$ decreasing ATPase activity, while in the case of SLN, the ATP activity is not affected, but the maximum calcium uptake rate is reduced, suggesting that there is a $\mathrm{Ca}^{2+}$ uncoupling function of SLN to SERCA [60]. Interestingly, SLN also plays an important role in regulating muscle thermogenesis. SLN mediates the uncoupling of $\mathrm{Ca}^{2+}$ ions and SERCA, which allows SERCA to execute a futile cycle of hydrolysis of ATP to ADP which generates heat without pumping $\mathrm{Ca}^{2+}$ ions, thus contributing to temperature homeostasis of the body, another critical function of muscle beyond contraction [49]. MLN function is still an emerging area of research, and recent studies suggest that MLN exerts an inhibitory regulation on SERCA activity; indeed, the genetic ablation of MLN was able to improve the SERCA function and enhance exercise performance [46]. In contrast, DWORF functions differently from the others in that it is an activator of SERCA. It has been reported that in cardiac muscle, genetic manipulation replacing PLN with DWORF results in a dramatic increase in SERCA activity and the muscle contractility is also improved [46].

\section{Age related in changes in SERCA activity}

Aging is a progressive process that is marked by a gradual compromise in biochemical and physiological capabilities resulting in reduced organ function. As a result, aging is the major risk factor for the development of cancer, neurodegenerative disorders, cardiovascular diseases, and neuromuscular myopathy [67]. A key cellular process associated with aging is oxidative stress, especially in skeletal muscle where a higher ROS production rate is associated with aged muscle $[40,55]$. In particular, oxidation and nitration of cellular protein has been suggested to be an underlying causal factor in the progressive loss of cellular functions [63]. Oxidative stress can exert a feedforward effect in which elevated oxidation of cellular components can further exacerbate the cellular oxidative stress environment and impair protein targets that are sensitive to oxidative inactivation. Unchecked, these protein modifications can promote mutations of nuclear and mitochondrial DNA (mtDNA), downregulating energy 
metabolism, generating more ROS and other harmful molecules, and further accumulating an even higher oxidative stress in aging cells $[17,63]$.

Oxidative stress impacts SERCA function and has consequent effects on skeletal muscle Commonly, oxidative stress is defined as the imbalance between the generation of pro-oxidants and antioxidant response, leading to an accumulation of oxidized molecules [9]. As mentioned above, aging has been associated with an increased oxidative stress, and in turn, the SERCA pump has been shown to be susceptible and sensitive to oxidative modifications. The first report that a reduced SERCA activity occurs in biological aging demonstrated a selective oxidation on cysteine residues of SERCA proteins [62]. More specifically, cysteine 674 was found to have an irreversible oxidation mediated by elevated hydrogen peroxide treatment. In cardiac muscle, oxidation on Cys674 dramatically reduced the SERCA activity and, importantly, impaired the cardiac myocyte relaxation in aged mouse heart. Genetic modification to replace the Cys674 with serine (Cys674Ser) resulted in a smaller degree of SERCA inactivation in response to oxidation by ROS [56]. In addition to cardiac muscle, Dremina et al. found cysteine residues in SERCA in skeletal muscle that can be modified by oxidation, including Cys674, Cys675 in SERCA 1, and Cys674 in SERCA 2 [12]. In skeletal muscle, these cysteine residues mediate the regulation of SERCA activity by reversible oxidation through peroxynitrite-induced glutathionylation, resulting in increased SERCA activity; however, excessive pathological oxidative stress will cause irreversible oxidation of cysteines including sulfonylation, causing reduced SERCA activity and impaired ability to regulate through glutathionylation [1]. Our group also reported previously that SERCA activity was impaired in skeletal muscle in a mouse model of high oxidative stress in response to a lack of CuZnSOD $(\operatorname{Sod} 1 \mathrm{KO})$. This is a genetically modified mouse model that exhibits a number of accelerated aging phenotypes including muscle atrophy and weakness [54]. Based on that study, we measured the SERCA activity in naturally aged muscles (26 months old mouse model) recently and also found evidence of increased oxidative stress, including increased ROS production along with dramatically impaired SERCA activity ( $41 \%$ loss) [55]. In response to oxidative inactivation of SERCA function, the calcium regulation in skeletal muscles may be impaired resulting in an elevated cytosolic $\mathrm{Ca}^{2+}$ concentration. This in turn has been reported to be detrimental to the excitation-contraction coupling (E-C coupling) system, as this system highly relies on the stable $\mathrm{Ca}^{2+}$ homeostasis balancing the $\mathrm{Ca}^{2+}$ release from ryanodine receptors (RyRs) during the contraction and the re-uptaking by SERCA during the relaxation $[3,7]$.
A defect in the EC coupling system will in turn compromise muscle functions, for example the decreased force generation, prolonged time to reach the peak force with the increased half relaxation time, and damaged the key calcium handling proteins in EC coupling pathway, i.e., ryanodine receptor, and all of these can eventually contribute to a final muscle weakness and sarcopenia [53]. In addition to affecting the EC coupling system, stable $\mathrm{Ca}^{2+}$ homeostasis is also important for mitochondria as $\mathrm{Ca}^{2+}$ signaling and the calcium concentration in mitochondria is critical for mitochondrial respiratory function [67]. For example, during ATP production, $\mathrm{Ca}^{2+}$ ions are required for activating the ATP synthase complex [66]; moreover, the activity of some dehydrogenases in the tricarboxylic acid cycle (TCA cycle) is also regulated by $\mathrm{Ca}^{2+}$ ions [20]. In physiological conditions, cytosolic $\mathrm{Ca}^{2+}$ signals are rapidly transduced into the mitochondrial matrix, although the uptake machinery in mitochondria has a lower affinity to $\mathrm{Ca}^{2+}$ relative to the SR [8]. Thus, an elevated cytosolic $\mathrm{Ca}^{2+}$ concentration caused by SERCA dysfunction can lead to altered mitochondrial function, negatively impacting cellular energy metabolism and even leading to cell death [8]. Mitochondria in a high cytosolic calcium environment in turn may produce more ROS, exacerbating the negative effect of oxidative stress on SERCA activity and contractile dysfunction in skeletal muscles, as we reported previously [2]. The potential associations between SERCA pumps and some critical cellular events in the face of an elevated oxidative stress are illustrated in Fig. 3. Because all of these events are closely associated with muscle metabolism and functions, dysfunctional SERCA pumps can influence muscle functions through a number of pathways.

The effects of neurodegenerative disorders and muscle disease on SERCA function Neurodegenerative disorders such as amyotrophic lateral sclerosis (ALS) alter neuromuscular transmission through the disruption of neuromuscular junction (NMJ) structures and function and are associated with a number of denervation phenotypes in skeletal muscles [35]. The NMJ has been shown to be a highly age-related structure with its morphology of the pre- and post-synaptic regions altered and the number of neurotransmitter-containing synaptic vesicles decreased $[24,59]$. The effects of NMJ dysfunction on skeletal muscles have been well reported, and SERCA activity has been reported to be dramatically reduced in denervated muscles. This could be due to the oxidative stress initiated by mitochondrial dysfunction and $\mathrm{LOOH}$ generation in response to denervation [28]; however, their exact interactions and underlying mechanisms are still not fully understood. Using the ALS mouse model (G93A), our group found that the loss of muscle innervation in this 


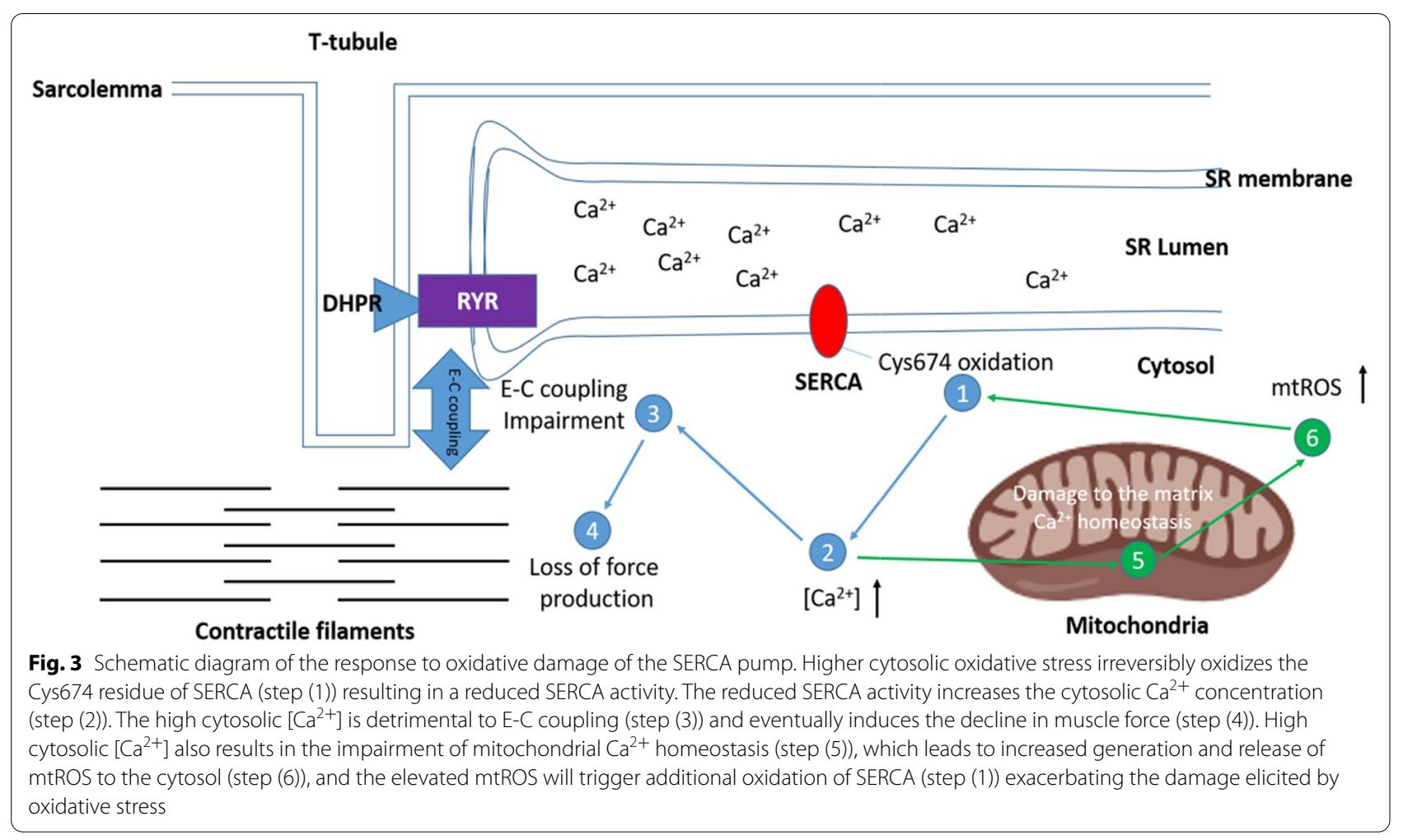

model was associated with a decrease in SERCA activity ( $\sim 20 \%$ decrease in muscle from G93A mice compared to wildtype mice), although the SERCA protein level was not reduced (This unpublished data was provided by Rizwan Qaisar \& Holly Van Remmen). In addition, in a recent study, we showed that overexpression of catalase targeted to skeletal muscle mitochondria (mMCAT) in the Sod 1 KO mouse muscle atrophy model led to reduced oxidative stress in muscle associated with full rescue of oxidative stress induced alterations in NMJ morphology and function, and SERCA activity is also returned to wildtype levels [70]. These studies support the close association between SERCA activity and muscle innervation status.

Other than neurodegenerative disorders, muscular dystrophies (MDs) are also a group of degenerative muscle disorders characterized by progressive muscle wasting and often premature death. Commonly, the primary defect to most MDs involves disruption of the dystrophin-glycoprotein complex (DGC), which leads to sarcolemma instability and an abnormal $\mathrm{Ca}^{2+}$ influx, inducing cellular necrosis $[16,19]$. Duchenne muscular dystrophy (DMD) is a severe form of MD that has been studied extensively using the dystrophic $m d x$ mouse model. In this model, SERCA activity is decreased more than $20 \%$ compared to wild type muscle in both hindlimb and diaphragm muscles. The impaired SERCA function contributes to the calcium dysregulation in dystrophic muscles [18], and enhancing the SERCA activity, i.e., by overexpression SERCA protein, suppresses the muscle degenerative phenotypes $[19,41]$. Together, these studies suggest an important role of SERCA function under the diseased status of skeletal muscle.

SERCA activity in muscle in response to denervation Currently, there are two potential factors that have been studied regarding the influence of denervation on SERCA function, one is through phospholamban (PLN) [28], and the other is via receptor activator nuclear factor-kB (RANK) $[14,15]$. As mentioned above, PLN is an inhibitor of SERCA pumps expressing in all muscle types. The data from Komatsu's study show that with denervation, the amount of PLN is elevated in all major fiber types, and as much as 3-fold in MHCIIx fibers [28]. Along with the increase of PLN, the SERCA activity, mainly in fast-twitch muscle, and the calcium content in the SR are found to be reduced, strongly suggesting that in this case of denervation, PLN is a major contributor to the inhibition of SERCA activity. It is noteworthy that the elevation of PLN in this study is due to a post-translational effect, i.e., they did not find any change in the mRNA level of 
PLN in skeletal muscles, suggesting that some potential post-translational pathways exert modifications on PLN. However, the post-translational regulation of PLN in skeletal muscle is still elusive, although some reports that have indicated PLN is degraded by proteasome or autophagy pathways in a ubiquitin-dependent manner in cardiac muscle $[45,65,71]$. Thus, it is possible that ubiquitin-dependent modifications play a role in PLN modification in skeletal muscle as well. It is possible that denervation of skeletal muscles might suppress ubiquitination of PLN by a mechanism such as down-regulation of E3 ubiquitin ligase specific to PLN leading to an increase in the amount of PLN [28].

Receptor-activator of nuclear factor-kB (RANK), its ligand RANKL, and the soluble decoy receptor osteoprotegerin (OPG) are members of the tumor necrosis factor superfamily that regulate bone remodeling [29, 32]. The study of the regulating roles of $\mathrm{Ca}^{2+}$ through the interactions between RANK and RANKL is still a newly emerging area, but its function related to regulating cellular $\mathrm{Ca}^{2+}$ storage and a series of $\mathrm{Ca}^{2+}$ pumps in denervated muscles has been measured [14, 15]. In a muscle-specific RANK knock out mouse model, they demonstrated that in denervated fast twitch muscles (EDL), the RANK deletion markedly increased the content of STIM 1 , a $\mathrm{Ca}^{2+}$ sensor, the SERCA activity, the SR $\mathrm{Ca}^{2+}$ storage, and also the corresponding muscular functions, such as force production and muscle fatigability [15]. This study established a novel role of RANK signaling in regulating $\mathrm{Ca}^{2+}$ homeostasis in skeletal muscles in responding to denervation. While this study is still preliminary and the longterm effects remain to be determined, these results open a new field of research and new therapeutic avenues for neuromuscular disease in aging.

\section{SERCA pumps as a potential therapeutic target} The effect of SERCA activation on skeletal muscle functions and neurodegenerative disorders

As discussed above, SERCA pumps are implicated in many diseases, and as a result targeting SERCA as a potential therapeutic strategy has attracted significant attention in recent years. Currently, there are two primary approaches for targeting the role of SERCA, one is to increase SERCA activity through stimulating SERCA by SERCA activators [57], and the other is to control SERCA expression through genetic manipulations [37].

There are a series of mechanisms that have been shown to activate SERCA, including glutathionylation [1, 34], SUMOylation [13, 22], acetylation [42], and allosteric activation [31], and a number of corresponding drugs have been well tested (Table. 2). For example, in skeletal muscle, our group recently tested the effects of a novel allosteric SERCA activator, CDN1163, on impaired muscle functions induced by high oxidative stress [54]. CDN1163 was firstly identified in a screen by the Kang group in an insulin resistance and type 2 diabetic model (ob/ob mice) [26]. By treating the ob/ob mice with CDN1163, along with the elevated SERCA activity, they found a number of beneficial alterations in metabolisms in these mice, including reduced adipose tissue, increased energy expenditure, attenuated ER stress response and ER stress-induced apoptosis, and improved mitochondrial biogenesis [26]. In our study, we tested the effectiveness of CDN1163 as an intervention to restoring the impaired SERCA function, and we reported in muscle of the CuZnSOD knock out (Sod1KO) mice. From our results, we found that CDN1163 is effective for restoring SERCA activity in Sod1KO mice without altering SERCA protein expression and that the restoration of SERCA activity prevented muscle atrophy in Sod1KO mice and in addition the contractile dysfunction is dramatically improved. Elevated SERCA activity in the muscle of the Sod1KO mice also was associated with reduced mitochondrial ROS generation suggesting an indirect

Table 2 Established mechanisms and tested drugs for stimulating SERCA via post-translational modifications or protein to protein interactions. The table lists the proved typical SERCA stimulating interactions, including glutathionylation, SUMOylation, acetylation and the activator through allosteric effect, and their corresponding regulating sites and effective drugs

\begin{tabular}{llll}
\hline $\begin{array}{l}\text { SERCA stimulating } \\
\text { interactions }\end{array}$ & Regulating sites & Effective drugs & Reference \\
\hline Glutathionylation & Disulfide bond formed at cysteine residue of SERCA & $\begin{array}{l}\text { Nitroxyl (HNO) } \\
\text { Nitric oxide (ONOO) }\end{array}$ & Adachi et al. [1], Lancel et al. [34] \\
SUMOylation & SUMO binds to lysine residue of SERCA & Luteolin \\
Acetylation & Addition of acetyl group at lysine residue on SERCA & $\begin{array}{l}\text { Suberanilohydroxamic acid } \\
\text { (HDAC inhibitor) }\end{array}$ & Du et al. [13], Hu et al. [22] \\
Allosteric activator & & CDN1 163 et al. [42]
\end{tabular}


beneficial effect of mitochondrial function [54]. In addition, our group further applied this activator, CDN1163, in aged mice (26 months old); similarly, it efficiently activated SERCA function and prevented muscle from atrophy and weakness at such an old age. In addition to these positive alterations on muscle, we also found beneficial changes in gene expression, for example, activation of AMPK signaling pathways for mitochondrial restoration and maintaining muscle mass, inhibition p38 MAPK pathway for reducing stress response, and also an upregulation of calcium related genes in responding to calcium signaling and handling. In summary, muscle atrophy and weakness are always coupled with reduced SERCA activity, and a defected SERCA function will then lead to increased ER/SR stress, which will in turn impair mitochondrial functions. Hence, as a newly emerging and promising intervention, restoration of SERCA activity by activators is efficient to improve muscle functions and metabolism and further mitigate sarcopenic phenotype.

The studies described above outline the effects of aging and muscle pathologies on SERCA in muscle and the potential for interventions that activate SERCA. In addition, the effects of neuronal SERCA stimulation on improving neurodegenerative disorders has also been investigated [57]. For instance, Alzheimer's disease (AD) is the most common neurodegenerative disease in elderly patients, and neuronal loss with the accumulation of $\beta$-amyloid peptide are the main features in $\mathrm{AD}$ neural tissues [23]. Amyloid precursor protein (APP) is a critical protein in the mechanisms underlying $\mathrm{AD}$, and the $A \beta$ fragments generated by the APP protein are the main constituents of the amyloid plaques in $\mathrm{AD}$ neural tissues [68]. It has been shown that the regulation of $A \beta$ production can be altered by changes in the homeostasis of the endoplasmic reticulum $\mathrm{Ca}^{2+}$ pool being a key point in this process [10, 27]. Parisiadou et al. have reported that the stimulation of neuronal SERCA can increase the ER $\mathrm{Ca}^{2+}$ store followed by the less influx of extracellular calcium to the cytosol of neuro cells, which in turn increases the interactions between APP and homer proteins, and hence reduces the accumulation of the amyloid plaques and further ameliorate the symptoms of $\mathrm{AD}[50]$.

\section{Genetic manipulations of SERCA in disease intervention}

The genetic manipulation of SERCA has been extensively studied using transgenic animals, but predominantly in cardiac tissue and with respect to heart function. The major strategy for genetically manipulating SERCA pumps has been to overexpress the specific SERCA isoforms (i.e., SERCA 2a) in certain tissues (i.e., cardiac muscle) to rebalance the calcium homeostasis $[43,51]$. Many studies used adenoviral (AAV-1) SERCA 2a as a gene tool to increase the expression of the SERCA 2a gene in animals or in human ventricular myocytes. Universally, these studies found that the overexpression of SERCA 2a isoforms efficiently increased the SERCA activity in cardiac tissues, and enhanced the contraction and relaxation velocity, and hence restored contractile function to normal levels [11, 21]. More recently, using AAV-1 mediated SERCA 2a gene transfer, it was found that the electrophysiological and mechanical functions were improved in the hearts from myocardial infarction (MI) models [43]. As for the skeletal muscle specific isoform SERCA 1, a popular way to overexpress SERCA 1 is to use a modified human skeletal muscle $\alpha$-actinin promotor to selectively increase its amount in fasttwitch muscles as described by Goonasekera et al. [19]. Some previous studies have indicated that the increased SERCA 1 expression in skeletal muscles improves calcium handling and muscle function and reduces markers of muscular damages in some muscular dystrophy mouse models, such as the mdx mice and the $\delta$-sarcoglycan-null $\left(\mathrm{Sgcd}^{-/-}\right)$mice $[19,41]$. Overall, all of these findings suggest that restoring $\mathrm{Ca}^{2+}$ transport by increasing SERCA levels via overexpression is effective for maintaining contractility and further improving the mechanical functions of muscles.

\section{Conclusions}

The SERCA pump plays a critical role in $\mathrm{Ca}^{2+}$ regulating. The proper function of SERCA is important in skeletal muscle metabolism. Substantial data has demonstrated that restoration or activation of SERCA activity through either stimulating the SERCA pumps or genetically manipulating the SERCA expressions may be an effective therapeutic approach for the treatment of some diseases, such as sarcopenia, cardiomyopathy, and some neurodegenerative disorders such as AD. Future studies targeting the underlying mechanisms of SERCA activity and its role in tissue metabolism and associated pathways are needed to further reveal a deep understanding of SERCA functions in various diseases conditions.

\footnotetext{
Abbreviations

SERCA: Sarcoendoplasmic reticulum calcium ATPase; SR: Sarcoendoplasmic reticulum; MD: Muscle dystrophy; PMCA: Plasma membrane $\mathrm{Ca}^{2+}$ ATPase; E-C coupling: Excitation-contraction coupling; RyR: Ryanodine receptor; MMPs: Matrix metalloproteinases; CICR: Calcium-induced calcium release; PLN: Phospholamban; SLN: Sarcolipin; DWORF: Dwarf open reading frame; MLN: Myoregulin; Sod 1KO: Copper zinc superoxide dismutase knock out; TCA cycle: Tricarboxylic acid cycle; ROS: Reactive oxygen species; ALS: Amyotrophic lateral sclerosis; NMJ: Neuromuscular junction; mMCAT: Skeletal muscle-specific mitochondrial catalase; DGC: Dystrophin-glycoprotein complex; DMD: Duchenne muscular dystrophy; RANK: Receptor activator nuclear factor-kB; OPG: Osteoprotegerin; EDL: Extensor digitorum longus; AD: Alzheimer's disease; APP: Amyloid precursor protein; AAV-1: Adenoviral; Sgcd ${ }^{-1-}: \delta$-sarcoglycan-null; Ml: Myocardial infarction.
} 


\section{Acknowledgements \\ Not applicable.}

\section{Authors' contributions}

HX and HVR conceived, designed, and drafted all sections of the manuscript. All authors read and approved the final draft of the manuscript.

\section{Funding}

This work was supported by a VA Merit grant (I01BX004453) and NIH grants NIA-AG051442 and NIA-AG050676. Dr. Van Remmen also receives salary support as a VA Senior Research Career Scientist award (IK6 BX005234).

\section{Availability of data and materials}

Data sharing is not applicable to this review, as no datasets were generated or analyzed in this article.

\section{Declarations}

\section{Ethics approval and consent to participate}

Not applicable.

\section{Consent for publication}

Not applicable.

\section{Competing interests}

The authors declare that they have no competing interests.

\section{Author details}

${ }^{1}$ Aging \& Metabolism Research Program, Oklahoma Medical Research Foundation, Oklahoma City, OK 73104, USA. ²Oklahoma City VA Medical Center, Oklahoma City, OK, USA. ${ }^{3}$ Department of Physiology, OUHSC, Oklahoma City, OK, USA.

Received: 5 May 2021 Accepted: 26 October 2021

Published online: 12 November 2021

\section{References}

1. Adachi T, Weisbrod RM, Pimentel DR, Ying J, Sharov VS, Schoneich C, et al. S-Glutathiolation by peroxynitrite activates SERCA during arterial relaxation by nitric oxide. Nat Med. 2004;10:1200-7.

2. Ahn B, Ranjit R, Premkumar P, Pharaoh G, Piekarz KM, Matsuzaki S, et al. Mitochondrial oxidative stress impairs contractile function but paradoxically increases muscle mass via fibre branching. J Cachexia Sarcopenia Muscle. 2019:10:411-28.

3. Ali S, Garcia JM. Sarcopenia, cachexia and aging: diagnosis, mechanisms and therapeutic options - a mini-review. Gerontology. 2014:60:294-305.

4. Anderson DM, Anderson KM, Chang CL, Makarewich CA, Nelson BR, McAnally JR, et al. A micropeptide encoded by a putative long noncoding RNA regulates muscle performance. Cell. 2015;160:595-606.

5. Berchtold MW, Brinkmeier H, Muntener M. Calcium ion in skeletal muscle: its crucial role for muscle function, plasticity, and disease. Physiol Rev. 2000;80:1215-65

6. Bers DM. Cardiac excitation-contraction coupling Nature. 2002;415:198-205.

7. Boncompagni S, d'Amelio L, Fulle S, Fano G, Protasi F. Progressive disorganization of the excitation-contraction coupling apparatus in aging human skeletal muscle as revealed by electron microscopy: a possible role in the decline of muscle performance. J Gerontol a-Biol. 2006:61:995-1008.

8. Bravo-Sagua R, Parra V, Lopez-Crisosto C, Diaz P, Quest AF, Lavandero S. Calcium transport and signaling in mitochondria. Compr Physiol. 2017:7:623-34.

9. Breitenbach M, Eckl P. Introduction to oxidative stress in biomedical and biological research. Biomolecules. 2015:5:1169-77.

10. Cheung KH, Shineman D, Muller M, Cardenas C, Mei L, Yang J, et al. Mechanism of $\mathrm{Ca} 2+$ disruption in Alzheimer's disease by presenilin regulation of InsP3 receptor channel gating. Neuron. 2008;58:871-83.
11. del Monte F, Harding SE, Schmidt U, Matsui T, Kang ZB, Dec GW, et al. Restoration of contractile function in isolated cardiomyocytes from failing human hearts by gene transfer of SERCA2a. Circulation. 1999;100:2308-11.

12. Dremina ES, Sharov VS, Davies MJ, Schoneich C. Oxidation and inactivation of SERCA by selective reaction of cysteine residues with amino acid peroxides. Chem Res Toxicol. 2007;20:1462-9.

13. Du YP, Liu P, Xu TD, Pan DF, Zhu H, Zhai NN, et al. Luteolin modulates SERCA2a leading to attenuation of myocardial ischemia/reperfusion injury via sumoylation at lysine 585 in mice. Cell Physiol Biochem. 2018:45:883-98.

14. Dufresne SS, Boulanger-Piette A, Bosse S, Frenette J. Physiological role of receptor activator nuclear factor-kB (RANK) in denervation-induced muscle atrophy and dysfunction. Receptors Clin Investig. 2016a;3:e13231-6.

15. Dufresne SS, Dumont NA, Boulanger-Piette A, Fajardo VA, Gamu D, KakeGuena SA, et al. Muscle RANK is a key regulator of Ca2+ storage, SERCA activity, and function of fast-twitch skeletal muscles. Am J Physiol-Cell Ph. 2016b:310:C663-72.

16. Emery AE. The muscular dystrophies. Lancet. 2002;359:687-95.

17. Finkel T, Holbrook NJ. Oxidants, oxidative stress and the biology of ageing. Nature. 2000;408:239-47.

18. Gehrig SM, van der Poel C, Sayer TA, Schertzer JD, Henstridge DC, Church $\mathrm{JE}$, et al. Hsp72 preserves muscle function and slows progression of severe muscular dystrophy. Nature. 2012;484:394-8.

19. Goonasekera SA, Lam CK, Millay DP, Sargent MA, Hajjar RJ, Kranias EG, et al. Mitigation of muscular dystrophy in mice by SERCA overexpression in skeletal muscle. J Clin Invest. 2011:121:1044-52.

20. Gunter TE, Buntinas L, Sparagna G, Eliseev R, Gunter K. Mitochondrial calcium transport: mechanisms and functions. Cell Calcium. 2000;28:285-96.

21. Hajjar RJ, Schmidt U, Matsui T, Guerrero JL, Lee KH, Gwathmey JK, et al. Modulation of ventricular function through gene transfer in vivo. P Nat Acad Sci USA. 1998;95:5251-6.

22. Hu W, Xu T, Wu P, Pan D, Chen J, Chen J, et al. Luteolin improves cardiac dysfunction in heart failure rats by regulating sarcoplasmic reticulum Ca(2+)-ATPase 2a. Sci Rep. 2017;7:41017.

23. Jack CR Jr, Knopman DS, Jagust WJ, Shaw LM, Aisen PS, Weiner MW, et al. Hypothetical model of dynamic biomarkers of the Alzheimer's pathological cascade. Lancet Neurol. 2010:9:119-28.

24. Jang YC, Van Remmen $\mathrm{H}$. Age-associated alterations of the neuromuscular junction. Exp Gerontol. 2011;46:193-8.

25. Janssen I, Heymsfield SB, Wang ZM, Ross R. Skeletal muscle mass and distribution in 468 men and women aged 18-88 yr. J Appl Physiol. 2000;89:81-8

26. Kang S, Dahl R, Hsieh W, Shin A, Zsebo KM, Buettner C, et al. Small molecular allosteric activator of the sarco/endoplasmic reticulum Ca2+-ATPase (SERCA) attenuates diabetes and metabolic disorders. J Biol Chem. 2016:291:5185-98.

27. King GD, Scott Turner R. Adaptor protein interactions: modulators of amyloid precursor protein metabolism and Alzheimer's disease risk? Exp Neurol. 2004;185:208-19.

28. Komatsu M, Nakada T, Kawagishi H, Kato H, Yamada M. Increase in phospholamban content in mouse skeletal muscle after denervation. J Muscle Res Cell Motil. 2018:39:163-73.

29. Kong YY, Feige U, Sarosi I, Bolon B, Tafuri A, Morony S, et al. Activated $T$ cells regulate bone loss and joint destruction in adjuvant arthritis through osteoprotegerin ligand. Nature. 1999;402:304-9.

30. Koubassova NA, Tsaturyan AK. Molecular mechanism of actin-myosin motor in muscle. Biochemistry-Moscow. 2011;76:1484-506.

31. Krajnak K, Dahl R. A new target for Alzheimer's disease: a small molecule SERCA activator is neuroprotective in vitro and improves memory and cognition in APP/PS1 mice. Bioorg Med Chem Lett. 2018;28:1591-4

32. Lacey DL, Timms E, Tan HL, Kelley MJ, Dunstan CR, Burgess T, et al. Osteoprotegerin ligand is a cytokine that regulates osteoclast differentiation and activation. Cell. 1998:93:165-76.

33. Lamb GD. Excitation-contraction coupling in skeletal muscle: comparisons with cardiac muscle. Clin Exp Pharmacol Physiol. 2000:27:216-24.

34. Lancel S, Zhang J, Evangelista A, Trucillo MP, Tong X, Siwik DA, et al. Nitroxyl activates SERCA in cardiac myocytes via glutathiolation of cysteine 674. Circ Res. 2009;104:720-3.

35. Larkin LM, Davis CS, Sims-Robinson C, Kostrominova TY, Van Remmen $\mathrm{H}$, Richardson A, et al. Skeletal muscle weakness due to deficiency of 
CuZn-superoxide dismutase is associated with loss of functional innervation. Am J Physiol-Reg I. 2011;301:R1400-7.

36. Lehnart SE, Maier LS, Hasenfuss G. Abnormalities of calcium metabolism and myocardial contractility depression in the failing heart. Heart Fail Rev. 2009;14:213-24

37. Liu L, Zhao W, Liu J, Gan Y, Liu L, Tian J. Epigallocatechin-3 gallate prevents pressure overload-induced heart failure by up-regulating SERCA2a via histone acetylation modification in mice. PLoS One. 2018;13:e0205123.

38. Lytton J, Westlin M, Burk SE, Shull GE, Maclennan DH. Functional comparisons between isoforms of the sarcoplasmic or endoplasmic-reticulum family of calcium pumps. J Biol Chem. 1992;267:14483-9.

39. MacLennan DH, Kranias EG. Phospholamban: a crucial regulator of cardiac contractility. Nat Rev Mol Cell Biol. 2003:4:566-77.

40. Mansouri A, Muller FL, Liu Y, Ng R, Faulkner J, Hamilton M, et al. Alterations in mitochondrial function, hydrogen peroxide release and oxidative damage in mouse hind-limb skeletal muscle during aging. Mech Ageing Dev. 2006;127:298-306.

41. Mazala DA, Pratt SJP, Chen D, Molkentin JD, Lovering RM, Chin ER. SERCA1 overexpression minimizes skeletal muscle damage in dystrophic mouse models. Am J Physiol Cell Physiol. 2015;308:C699-709.

42. Meraviglia V, Bocchi L, Sacchetto R, Florio MC, Motta BM, Corti C, et al. HDAC inhibition improves the sarcoendoplasmic reticulum $\mathrm{Ca}(2+)$ ATPase activity in cardiac myocytes. Int J Mol Sci. 2018;19.

43. Motloch LJ, Cacheux M, Ishikawa K, Xie C, Hu J, Aguero J, et al. Primary effect of SERCA 2a gene transfer on conduction reserve in chronic myocardial infarction. J Am Heart Assoc. 2018;7:e009598.

44. Murphy RM, Verburg E, Lamb GD. Ca2+ activation of diffusible and bound pools of mu-calpain in rat skeletal muscle. J Physiol. 2006;576:595-612.

45. Nakagawa T, Yokoe S, Asahi M. Phospholamban degradation is induced by phosphorylation-mediated ubiquitination and inhibited by interaction with cardiac type Sarco(endo)plasmic reticulum Ca2+-ATPase. Biochem Bioph Res Co. 2016:472:523-30.

46. Nelson BR, Makarewich CA, Anderson DM, Winders BR, Troupes CD, Wu FF, et al. A peptide encoded by a transcript annotated as long noncoding RNA enhances SERCA activity in muscle. Science. 2016;351:271-5.

47. Olesen C, Picard M, Winther AM, Gyrup C, Morth JP, Oxvig C, et al. The structural basis of calcium transport by the calcium pump. Nature. 2007:450:1036-42.

48. Olesen C, Sorensen TL, Nielsen RC, Moller JV, Nissen P. Dephosphorylation of the calcium pump coupled to counterion occlusion. Science. 2004;306:2251-5.

49. Pant M, Bal NC, Periasamy M. Sarcolipin: a key thermogenic and metabolic regulator in skeletal muscle. Trends Endocrin Met. 2016;27:881-92.

50. Parisiadou L, Bethani I, Michaki V, Krousti K, Rapti G, Efthimiopoulos S. Homer2 and Homer3 interact with amyloid precursor protein and inhibit Abeta production. Neurobiol Dis. 2008;30:353-64.

51. Periasamy M, Kalyanasundaram A. SERCA pump isoforms: their role in calcium transport and disease. Muscle Nerve. 2007;35:430-42.

52. Periasamy M, Maurya SK, Sahoo SK, Singh S, Sahoo SK, Reis FCG, et al. Role of SERCA pump in muscle thermogenesis and metabolism. Compr Physiol. 2017;7:879-90.

53. Qaisar R, Bhaskaran S, Premkumar P, Ranjit R, Natarajan KS, Ahn B, et al. Oxidative stress-induced dysregulation of excitation-contraction coupling contributes to muscle weakness. J Cachexia Sarcopenia Muscle. 2018;9:1003-17.

54. Qaisar R, Bhaskaran S, Ranjit R, Sataranatarajan K, Premkumar P, Huseman $\mathrm{K}$, et al. Restoration of SERCA ATPase prevents oxidative stress-related muscle atrophy and weakness. Redox Biol. 2019;20:68-74.
55. Qaisar R, Pharaoh G, Bhaskaran S, Xu H, Ranjit R, Bian J, et al. Restoration of sarcoplasmic reticulum $\mathrm{Ca}(2+)$ ATPase (SERCA) activity prevents agerelated muscle atrophy and weakness in mice. Int J Mol Sci. 2020;22.

56. Qin F, Siwik DA, Lancel S, Zhang J, Kuster GM, Luptak I, et al. Hydrogen peroxide-mediated SERCA cysteine 674 oxidation contributes to impaired cardiac myocyte relaxation in senescent mouse heart. J Am Heart Assoc. 2013;2:e000184.

57. Rahate K, Bhatt LK, Prabhavalkar KS. SERCA stimulation: a potential approach in therapeutics. Chem Biol Drug Des. 2020;95:5-15.

58. Ren X, Lamb GD, Murphy RM. Distribution and activation of matrix metalloproteinase-2 in skeletal muscle fibers. Am J Physiol Cell Physiol. 2019;317:C613-25.

59. Rygiel KA, Picard M, Turnbull DM. The ageing neuromuscular system and sarcopenia: a mitochondrial perspective. J Physiol. 2016;594:4499-512.

60. Sahoo SK, Shaikh SA, Sopariwala DH, Bal NC, Periasamy M. Sarcolipin protein interaction with Sarco(endo) plasmic Reticulum Ca2+ ATPase (SERCA) is distinct from phospholamban protein, and only sarcolipin can promote uncoupling of the SERCA pump. J Biol Chem. 2013;288:6881-9.

61. Shaikh SA, Sahoo SK, Periasamy M. Phospholamban and sarcolipin: are they functionally redundant or distinct regulators of the Sarco(Endo) Plasmic Reticulum Calcium ATPase? J Mol Cell Cardiol. 2016;91:81-91.

62. Sharov VS, Dremina ES, Galeva NA, Williams TD, Schoneich C. Quantitative mapping of oxidation-sensitive cysteine residues in SERCA in vivo and in vitro by HPLC-electrospray-tandem MS: selective protein oxidation during biological aging. Biochem J. 2006;394:605-15.

63. Squier TC. Oxidative stress and protein aggregation during biological aging. Exp Gerontol. 2001;36:1539-50.

64. Sweadner KJ, Donnet C. Structural similarities of Na,K-ATPase and SERCA, the Ca2+-ATPase of the sarcoplasmic reticulum. Biochem J. 2001;356:685-704.

65. Teng AC, Miyake T, Yokoe S, Zhang L, Rezende LM Jr, Sharma P, et al. Metformin increases degradation of phospholamban via autophagy in cardiomyocytes. Proc Natl Acad Sci U S A. 2015;1 12:7165-70.

66. Territo PR, Mootha VK, French SA, Balaban RS. Ca(2+) activation of heart mitochondrial oxidative phosphorylation: role of the $F(0) / F(1)$-ATPase. Am J Physiol Cell Physiol. 2000;278:C423-35.

67. Ureshino RP, Rocha KK, Lopes GS, Bincoletto C, Smaili SS. Calcium signaling alterations, oxidative stress, and autophagy in aging. Antioxid Redox Signal. 2014;21:123-37.

68. van der Kant R, Goldstein LS. Cellular functions of the amyloid precursor protein from development to dementia. Dev Cell. 2015;32:502-15.

69. Watson H. Biological membranes. Essays Biochem. 2015:59:43-69.

70. Xu H, Ranjit R, Richardson A, Van Remmen H. Muscle mitochondrial catalase expression prevents neuromuscular junction disruption, atrophy, and weakness in a mouse model of accelerated sarcopenia. J Cachexia Sarcopenia Muscle. 2021.

71. Yokoe S, Asahi M. Phospholamban is downregulated by pVHL-mediated degradation through oxidative stress in failing heart. Int J Mol Sci. 2017:18.

\section{Publisher's Note}

Springer Nature remains neutral with regard to jurisdictional claims in published maps and institutional affiliations.

Ready to submit your research? Choose BMC and benefit from:

- fast, convenient online submission

- thorough peer review by experienced researchers in your field

- rapid publication on acceptance

- support for research data, including large and complex data types

- gold Open Access which fosters wider collaboration and increased citations

- maximum visibility for your research: over $100 \mathrm{M}$ website views per year

At BMC, research is always in progress.

Learn more biomedcentral.com/submissions 